\title{
Update on cesarean delivery and small newborn for gestational age
}

\section{Atualização em CeSARIANA e RECÉM-NASCIDO PEQUENo PARA A IDADE GESTACIONAL}

Ricardo Simões ${ }^{1,2}$, Wanderley M. Bernardo ${ }^{2}$, Romulo P. Soares ${ }^{2}$, Antônio J. Salomão ${ }^{1}$, Edmund C. Baracat ${ }^{1}$

${ }^{1}$ Federacão Brasileira das Associaç̃oes de Ginecologia e Obstetrícia (Febrasgo)

${ }^{2}$ Programa Diretrizes da AMB

http://dx.doi.org/10.1590/1806-9282.62.01.14

1. What is the most important single factor in neonatal mortality, in addition to being a significant determinant of postnatal mortality and infant morbidity?

a. Muscle tone at birth.

b. Gestational age.

c. Apgar score.

d. Birth weight.

2. A retrospective study considered in this analysis suggested that SGA newborns exposed to labor have increased risk of early neonatal death compared with those not subject to labor. But in the same study, a lower risk of death was identified for what periods?

a. For the late neonatal and postnatal periods in those born vaginally, regardless of gestational age.

b. For the late neonatal period in those born vaginally, regardless of gestational age.

c. For the early neonatal and postnatal periods in those born by cesarean section, regardless of gestational age.

d. For the early neonatal period in those born by cesarean section, regardless of gestational age.

3. A retrospective study by Lee HC et al. showed contradictory results; what was found?

a. SGA newborns with 24 weeks presented a decrease in early neonatal mortality at the time of vaginal delivery, while SGA newborns with gestational age > 33 weeks, as well as those regarded as AGA (appropriate for gestational age), showed an increase in mortality in cases of vaginal delivery.

b. SGA newborns with 31 weeks presented a decrease in late neonatal mortality at the time of cesarean section, while SGA newborns with gestational age > 33 weeks, as well as those regarded as AGA (appropriate for gestational age), showed an increase in mortality in cases of cesarean section. c. SGA newborns with 24 weeks presented a decrease in early neonatal mortality at the time of vaginal delivery, while SGA newborns with gestational age > 36 weeks, as well as those regarded as AGA (appropriate for gestational age), showed an increase in mortality in cases of vaginal delivery.

d. SGA newborns with 28 weeks presented a decrease in late neonatal mortality at the time of cesarean section, while SGA newborns with gestational age > 36 weeks, as well as those regarded as AGA (appropriate for gestational age), showed an increase in mortality in cases of cesarean section.

4. According to Boers KE et al., was there any difference in neonatal morbidity and mortality, as assessed by Apgar score, among fetuses born vaginally or undergoing elective cesarean delivery?

a. There was a significant difference of $3 \%$ in neonatal morbidity and mortality.

b. There was a significant difference of $5 \%$ in neonatal morbidity and mortality.

c. There was no difference in neonatal morbidity and mortality.

d. There was a significant difference of $7 \%$ in neonatal morbidity and mortality.

5. What can we say with regard to mode of delivery of small for gestational age fetuses in order to reduce neonatal and perinatal morbidity and mortality?

a. In the absence of other obstetric indications requiring cesarean section, there is sufficient evidence to recommend early cesarean section in the pregnancy of small for gestational age fetuses to reduce neonatal and perinatal morbidity and mortality.

b. In the absence of other obstetric indications requiring cesarean section, there is sufficient evidence to recommend early cesarean section in the pregnancy of small for gestational age fetuses to reduce neonatal mortality only. 
c. In the absence of other obstetric indications requiring cesarean section, there is no sufficient evidence to recommend planned cesarean section in the pregnancy of small for gestational age fetuses aiming to reduce neonatal and perinatal morbidity and mortality.

d. In the absence of other obstetric indications requiring cesarean section, there is sufficient evidence to recommend early cesarean section in the pregnancy of small for gestational age fetuses to reduce perinatal morbidity.

\section{ANSWERS TO CLINICAL SCENARIO: UPDATE ON CESAREAN DELIVERY AND PREMATURITY [PUBLISHED IN RAMB 2015; 61(6)]}

1. The indication of cesarean delivery in preterm pregnancy can be based on three proposals. They include:

All of the above are correct. (Alternative D)
2. Cesarean section in preterm pregnancy also presents a particular problem related to surgical technique, because:

The lower segment may not be formed. (Alternative B)

3. Regarding the fetal trauma at birth and maternal outcomes, it is true that:

There is no difference in fetal trauma between cesarean section and vaginal delivery. (Alternative $\mathbf{C}$ )

4. The concept of planned cesarean section in preterm deliveries implies:

Accurately diagnosing, and performing a C-section early in the period of labor, or right before it. (Alternative A)

5. Regarding cesarean delivery and prematurity, the recommendation is:

Planned cesarean section in preterm fetuses in cephalic presentation should not be indicated with the purpose of fetal protection. (Alternative B) 